\title{
A Real Representation Method for Solving Yakubovich-j-Conjugate Quaternion Matrix Equation
}

\author{
Caiqin Song, ${ }^{1,2}$ Jun-e Feng, ${ }^{1}$ Xiaodong Wang, ${ }^{3}$ and Jianli Zhao ${ }^{4}$ \\ ${ }^{1}$ School of Mathematics, Shandong University, Jinan 250100, China \\ ${ }^{2}$ College of Information Science and Engineering, Shandong University of Science and Technology, Qingdao 266590, China \\ ${ }^{3}$ School of Astronautics, Harbin Institute of Technology, Harbin 150001, China \\ ${ }^{4}$ College of Mathematics Science, Liaocheng University, Liaocheng 252059, China
}

Correspondence should be addressed to Caiqin Song; songcaiqin1983@163.com and Jun-e Feng; fengjune@sdu.edu.cn

Received 19 October 2013; Revised 12 December 2013; Accepted 14 December 2013; Published 12 January 2014

Academic Editor: Ngai-Ching Wong

Copyright (C) 2014 Caiqin Song et al. This is an open access article distributed under the Creative Commons Attribution License, which permits unrestricted use, distribution, and reproduction in any medium, provided the original work is properly cited.

\begin{abstract}
A new approach is presented for obtaining the solutions to Yakubovich-j-conjugate quaternion matrix equation $X-A \widehat{X} B=C Y$ based on the real representation of a quaternion matrix. Compared to the existing results, there are no requirements on the coefficient matrix $A$. The closed form solution is established and the equivalent form of solution is given for this Yakubovich- $j$ conjugate quaternion matrix equation. Moreover, the existence of solution to complex conjugate matrix equation $X-A \bar{X} B=C Y$ is also characterized and the solution is derived in an explicit form by means of real representation of a complex matrix. Actually, Yakubovich-conjugate matrix equation over complex field is a special case of Yakubovich- $j$-conjugate quaternion matrix equation $X-A \widehat{X} B=C Y$. Numerical example shows the effectiveness of the proposed results.
\end{abstract}

\section{Introduction}

The linear matrix equation $X-A X B=C$, which is called the Kalman-Yakubovich matrix equation in [1], is closely related to many problems in conventional linear control systems theory, such as pole assignment design [2], Luenberger-type observer design [3, 4], and robust fault detection [5, 6]. In recent years, many studies have been reported on the solutions to many algebraic equations including quaternion matrix equations and nonlinear matrix equations. Yuan and Liao [7] investigated the least squares solution of the quaternion $j$-conjugate matrix equation $X-A \widehat{X} B=C$ (where $\widehat{X}$ denotes the $j$-conjugate of quaternion matrix $X$ ) with the least norm using the complex representation of quaternion matrix, the Kronecker product of matrices, and the MoorePenrose generalized inverse. The authors in [8] considered the matrix nearness problem associated with the quaternion matrix equation $A X A^{H}+B Y B^{H}=C$ by means of the CCD-Q, GSVD-Q, and the projection theorem in the finite dimensional inner product space. In addition, Song et al.
$[9,10]$ established the explicit solutions to the quaternion $j$ conjugate matrix equation $X-A \widehat{X} B=C, X F-A \widehat{X}=C Y$, but here the known quaternion matrix $A$ is a block diagonal form. Wang et al. in $[11,12]$ investigated Hermitian tridiagonal solutions and the minimal-norm solution with the least norm of quaternionic least squares problem in quaternionic quantum theory. Besides, in $[13,14]$, some solutions for the Kalman-Yakubovich equation are presented in terms of the coefficients of characteristic polynomial of matrix $A$ or the Leverrier algorithm. The existence of solution to the matrix equation $X-A \bar{X} B=C$, which, for convenience, is called the Kalman-Yakubovich-conjugate matrix equation, is established, and the explicit solution is derived. Several necessary and sufficient conditions for the existence of a unique solution to the matrix equation $\sum_{i=0}^{k} A^{i} X B_{i}=E$ over quaternion field are obtained [15]. The authors in [1618] have provided the consistence of the matrix equation $A X-\bar{X} B=C$ via the consimilarity of two matrices. In [19], $\mathrm{Wu}$ et al. construct some explicit expressions of the solution of the matrix equation $A X-\bar{X} B=C$ by means of a real 
representation of a complex matrix. It is shown that there exists a unique solution if and only if $A \bar{A}$ and $B \bar{B}$ have no common eigenvalues.

In this paper, we study quaternion $j$-conjugate matrix equation $X-A \widehat{X} B=C Y$ by means of real representation of a quaternion matrix. Compared to the complex representation method $[9,10]$, the real representation method does not require any special case of the known matrix $A$. We propose the explicit solutions to the above Yakubovich- $j$-conjugate quaternion matrix equation. As the special case of quaternion j-conjugate matrix equation $X-A \widehat{X} B=C Y$, complex conjugate matrix equation $X-A \bar{X} B=C$ and Kalman-Yakubovich quaternion matrix equation are also investigated. The explicit solutions to the complex conjugate matrix equation have been established.

Throughout this paper, we use the following notations. Let $R$ denote the real number field, $C$ the complex number field, and $Q=R \oplus R i \oplus R j \oplus R k$ the quaternion field, where $i^{2}=j^{2}=k^{2}=-1, i j=-j i=k \cdot R^{m \times n}\left(C^{m \times n}\right.$ or $\left.Q^{m \times n}\right)$ denotes the set of all $m \times n$ matrices on $R(C$ or $Q)$. For any matrix $A \in C^{m \times n}, A^{T}, \bar{A}, A^{H}$, $\operatorname{det} A$, and $A^{*}$ represent the transpose, conjugate, conjugate transpose, determinant, and adjoint of $A$, respectively. In addition, symbol $A_{\sigma}$ is the real representation of quaternion matrix $A . A \otimes B=\left(a_{i j} B\right)$ denotes the Kronecker product of two matrices $A$ and $B$. If $A \in Q^{m \times n}$, let $A=A_{1}+A_{2} i+A_{3} j+A_{4} k$, where $A_{t} \in R^{m \times n}, t=$ $1, \ldots, 4$, and define $\widehat{A}=A_{1}-A_{2} i+A_{3} j-A_{4} k$ to be the $j$ conjugate of $A$. For $A \in C^{m \times n}, \operatorname{vec}(A)$ is defined as $\operatorname{vec}(A)=$ $\left[\begin{array}{llll}a_{1}^{T} & a_{2}^{T} & \cdots & a_{n}^{T}\end{array}\right]^{T}$. Furthermore, letting $A \in Q^{n \times n}, B \in Q^{n \times r}$, and $C \in Q^{m \times n}$, we have the following notations associated with these matrices:

$$
\begin{aligned}
& Q_{c}(A, B, n)=\left[\begin{array}{llll}
B & A B & \cdots & A^{n-1} B
\end{array}\right], \\
& Q_{o}(A, C, k)=\left[\begin{array}{c}
C \\
C A \\
\vdots \\
C A^{k-1}
\end{array}\right], \\
& f_{A_{\sigma}}(s)=\operatorname{det}\left(s I-A_{\sigma}\right)=s^{2 n}+\alpha_{2 n-1} s^{2 n-1}+\cdots+\alpha_{1} s+\alpha_{0} \text {, } \\
& S_{r}\left(I, A_{\sigma}\right)=\left[\begin{array}{ccccc}
I_{r} & \alpha_{2} I_{r} & \alpha_{4} I_{r} & \cdots & \alpha_{2(n-1)} I_{r} \\
& I_{r} & \alpha_{2} I_{r} & \cdots & \alpha_{2(n-2)} I_{r} \\
& & & \cdots & \cdots \\
& & & I_{r} & \alpha_{2} I_{r} \\
& & & & I_{r}
\end{array}\right]
\end{aligned}
$$

Obviously, $Q_{c}(A, B, n)$ is the controllability matrix of the matrix pair $(A, B), Q_{o}(A, C, k)$ is the observability matrix of the matrix pair $(A, C)$, and $S_{r}\left(I, A_{\sigma}\right)$ is a symmetric matrix.

\section{Quaternion-j-Conjugate Matrix Equation

$$
X-A \widehat{X} B=C Y
$$

2.1. Real Matrix Equation $X-A X B=C Y$. In this subsection, we investigate the Yakubovich matrix equation over real field

$$
X-A X B=C Y \text {. }
$$

Theorem 1. Suppose the real matrices $A \in R^{n \times n}, B \in R^{p \times p}$, $C \in R^{n \times r},\{s \mid \operatorname{det}(I-s A)=0\} \cap \lambda(B)=\phi$; let

$$
\begin{gathered}
f_{(I, A)}(s)=\operatorname{det}(I-s A)=\alpha_{n} s^{n}+\cdots+\alpha_{1} s+\alpha_{0}, \quad \alpha_{0}=1, \\
\operatorname{adj}(I-s A)=R_{n-1} s^{n-1}+\cdots+R_{1} s+R_{0} .
\end{gathered}
$$

Then, all the solutions to the Yakubovich matrix equation (2) can be established as

$$
\begin{aligned}
& X=\sum_{i=0}^{n-1} R_{i} C Z B^{i}, \\
& Y=Z f_{(I, A)}(B),
\end{aligned}
$$

where the matrix $Z \in R^{r \times p}$ is an arbitrary matrix.

Proof. We first show that the matrices $X$ and $Y$ given in (4) are solutions of the matrix equation (2). By the direct calculation we have

$$
\begin{aligned}
X-A X B= & \sum_{i=0}^{n-1} R_{i} C Z B^{i}-A \sum_{i=0}^{n-1} R_{i} C Z B^{i} B \\
= & \sum_{i=0}^{n-1} R_{i} C Z B^{i}-\sum_{i=0}^{n-1} A R_{i} C Z B^{i+1} \\
= & R_{0} C Z+\sum_{i=1}^{n-1}\left(R_{i}-A R_{i-1}\right) C Z B^{i} \\
& -A R_{n-1} C Z B^{n} .
\end{aligned}
$$

Due to the relation $(I-s A) \operatorname{adj}(I-s A)=I \operatorname{det}(I-s A)$, it is easily derived that

$$
\begin{gathered}
R_{0}=\alpha_{0} I, \\
R_{i}-A R_{i-1}=\alpha_{i} I, \quad i=1: n-1, \\
-A R_{n-1}=\alpha_{n} I .
\end{gathered}
$$

So one has

$$
\begin{gathered}
R_{0} C Z+\sum_{i=1}^{n-1}\left(R_{i}-A R_{i-1}\right) C Z B^{i}-A R_{n-1} C Z B^{n} \\
=C Z \sum_{i=0}^{n} \alpha_{i} B^{i}=C Z f_{(I, A)}(B)=C Y .
\end{gathered}
$$

Thus, the matrices $X$ and $Y$ given in (4) satisfy the matrix equation (2).

Secondly, we show the completeness of solution (4). It follows from Theorem 6 of [20] that there are $r p$ degrees of freedom in the solution of matrix equation (2), while solution (4) has exactly $r p$ parameters represented by the elements of the free matrix $Z$. Therefore, in the following we only need to show that all the parameters in the matrix $Z$ contribute to the solution. To do this, it suffices to show that the mapping $Z \rightarrow(X, Y)$ defined by (5) is injective. This is true since $f_{(I, A)}(B)$ is nonsingular under the condition of $\{s \mid \operatorname{det}(I-$ $s A)=0\} \cap \lambda(B)=\phi$. The proof is thus completed. 
In [21], we can find the following well-known generalized Faddeev-Leverrier algorithm:

$$
\begin{array}{ll}
R_{k}=R_{k-1} A+\alpha_{k} I_{n}, & R_{0}=I_{n}, \quad k=1,2, \ldots, n, \\
\alpha_{k}=\frac{\operatorname{trace}\left(R_{k-1} A\right)}{k}, & \alpha_{0}=1, \quad k=1,2, \ldots, n,
\end{array}
$$

where $\alpha_{i}, i=0,1,2, \ldots, n-1$, are the coefficients of the characteristic polynomial of the matrix $A$, and $R_{i}, i=$ $0,1, \ldots, n-1$, are the coefficient matrices of the adjoint matrix $\operatorname{adj}\left(s I_{n}-A\right)$.

Theorem 2. Given matrices $A \in R^{n \times n}, B \in R^{p \times p}, C \in R^{r \times p}$, let

$$
\begin{aligned}
f_{(I, A)}(s)= & \operatorname{det}(I-s A)=\alpha_{n} s^{n} \\
& +\cdots+\alpha_{1} s+\alpha_{0}, \quad \alpha_{0}=1 .
\end{aligned}
$$

Then the matrices $X$ and $Y$ given by (4) have the following equivalent form:

$$
\begin{gathered}
X=\sum_{j=0}^{n-1} \sum_{k=0}^{j} \alpha_{k} A^{j-k} C Z B^{j}, \\
Y=Z f_{(I, A)}(B) .
\end{gathered}
$$

Proof. According to (8), the following is easily obtained:

$$
\begin{gathered}
R_{0}=I, \\
R_{1}=\alpha_{1} I+A, \\
R_{2}=\alpha_{2} I+\alpha_{1} A+A^{2}, \\
\vdots \\
R_{n-1}=\alpha_{n-1} I+\alpha_{n-2} A+\cdots+A^{n-1} .
\end{gathered}
$$

This relation can be compactly expressed as

$$
R_{j}=\sum_{k=0}^{j} \alpha_{k} A^{j-k}, \quad \alpha_{0}=1, \quad j=1,2, \ldots, n-1 .
$$

Substituting this into the expression of $X$ in (10) and recording the sum, we have

$$
\begin{aligned}
X & =\sum_{j=0}^{n-1} R_{j} C Z B^{j}=\sum_{j=0}^{n-1}\left(\sum_{k=0}^{j} \alpha_{k} A^{j-k}\right) C Z B^{j} \\
& =\sum_{j=0}^{n-1} \sum_{k=0}^{j} \alpha_{k} A^{j-k} C Z B^{j} .
\end{aligned}
$$

Combining this with Theorem 1 gives the conclusion.

2.2. Real Representation of a Quaternion Matrix. For any quaternion matrix $A=A_{1}+A_{2} i+A_{3} j+A_{4} k \in Q^{m \times n}$,
$A_{l} \in R^{m \times n}(l=1,2,3,4)$, the real representation matrix of quaternion matrix $A$ can be defined as

$$
A_{\sigma}=\left[\begin{array}{cccc}
A_{1} & A_{2} & -A_{3} & A_{4} \\
A_{2} & -A_{1} & -A_{4} & -A_{3} \\
A_{3} & -A_{4} & A_{1} & A_{2} \\
A_{4} & A_{3} & A_{2} & -A_{1}
\end{array}\right] \in R^{4 m \times 4 n}
$$

For a $m \times n$ quaternion matrix $A$, we define $A_{\sigma}^{t}=\left(A_{\sigma}\right)^{t}$. In addition, if we let

$$
\begin{array}{rlrl}
P_{t} & =\left[\begin{array}{cccc}
I_{t} & 0 & 0 & 0 \\
0 & -I_{t} & 0 & 0 \\
0 & 0 & I_{t} & 0 \\
0 & 0 & 0 & -I_{t}
\end{array}\right], & Q_{t}=\left[\begin{array}{cccc}
0 & -I_{t} & 0 & 0 \\
I_{t} & 0 & 0 & 0 \\
0 & 0 & 0 & I_{t} \\
0 & 0 & -I_{t} & 0
\end{array}\right], \\
S_{t}=\left[\begin{array}{cccc}
0 & 0 & 0 & -I_{t} \\
0 & 0 & I_{t} & 0 \\
0 & -I_{t} & 0 & 0 \\
I_{t} & 0 & 0 & 0
\end{array}\right], & R_{t}=\left[\begin{array}{cccc}
0 & 0 & I_{t} & 0 \\
0 & 0 & 0 & I_{t} \\
-I_{t} & 0 & 0 & 0 \\
0 & -I_{t} & 0 & 0
\end{array}\right],
\end{array}
$$

in which $I_{t}$ is a $t \times t$ identity matrix, then $P_{t}, Q_{t}, S_{t}, R_{t}$ are unitary matrices.

The real representation has the following properties, which are given in [13].

Proposition 3. Let $A, B \in Q^{m \times n}, C \in Q^{n \times s}, a \in R$. Then

(1) $(A+B)_{\sigma}=A_{\sigma}+B_{\sigma},(a A)_{\sigma}=a A_{\sigma},(A C)_{\sigma}=A_{\sigma} P_{n} C_{\sigma}=$ $A_{\sigma}(\widehat{C})_{\sigma} P_{s}$

(2) $A=B \Leftrightarrow A_{\sigma}=B_{\sigma}$;

(3) $Q_{m}^{-1} A_{\sigma} Q_{n}=-A_{\sigma}, R_{m}^{-1} A_{\sigma} R_{n}=A_{\sigma}, S_{m}^{-1} A_{\sigma} S_{n}=-A_{\sigma}$, $P_{m}^{-1} A_{\sigma} P_{n}=(\widehat{A})_{\sigma} ;$

(4) the quaternion matrix $A$ is nonsingular if and only if $A_{\sigma}$ is nonsingular, and the quaternion matrix $A$ is an unitary matrix if and only if $A_{\sigma}$ is an orthogonal matrix;

(5) if $A \in Q^{m \times m}$, then $A_{\sigma}^{2 k}=\left((A \widehat{A})^{k}\right)_{\sigma} P_{m}$;

(6) $A \in Q^{m \times m}, B \in Q^{n \times n}, C \in Q^{m \times n}$, and $k+l$ is even, then

$A_{\sigma}^{k} C_{\sigma} B_{\sigma}^{l}$

$$
= \begin{cases}\left((A \widehat{A})^{s}(A \widehat{C} B)(\widehat{B} B)^{t}\right)_{\sigma}, & k=2 s+1, l=2 t+1, \\ \left((A \widehat{A})^{s} C(\widehat{B} B)^{t}\right)_{\sigma}, & k=2 s, l=2 t .\end{cases}
$$

Proposition 4. If $\lambda$ is a characteristic value of $A_{\sigma}$, then so are $\pm \lambda, \pm \bar{\lambda}$.

For any $A \in Q^{m \times m}$, let the characteristic polynomial of the real representation matrix $A_{\sigma}$ be $f_{\left(I, A_{\sigma}\right)}(\lambda)=\operatorname{det}\left(I_{4 m}-\right.$ $\left.\lambda A_{\sigma}\right)=\sum_{k=0}^{2 m} a_{2 k} \lambda^{2 k}$, and define $h_{A_{\sigma}}(\lambda)=\lambda^{4 m} f_{\left(I, A_{\sigma}\right)}\left(\lambda^{-1}\right)=$ $\sum_{k=0}^{2 m} a_{2 k} \lambda^{2(2 m-k)}$. So by Propositions 3 and 4 we have the following proposition. 
Proposition 5. Let $A \in Q^{m \times m}, B \in Q^{n \times n}$. Then

(1) $f_{\left(I, A_{\sigma}\right)}(\lambda)$ is a real polynomial, and $f_{\left(I, A_{\sigma}\right)}(\lambda)=$ $\sum_{k=0}^{2 m} a_{2 k} \lambda^{2 k}$

(2) $h_{A_{\sigma}}(\lambda)$ is a real polynomial, and $h_{A_{\sigma}}(\lambda)=$ $\sum_{k=0}^{2 m} a_{2 k} \lambda^{2(2 m-k)}$

(3) $h_{A_{\sigma}}\left(B_{\sigma}\right)=\left(g_{A_{\sigma}}(B \widetilde{B})\right)_{\sigma} P_{n}, f_{\left(I, A_{\sigma}\right)}\left(B_{\sigma}\right)=\left(p_{A_{\sigma}}(B \widetilde{B})\right)_{\sigma}$ $P_{n}$, in which $g_{A_{\sigma}}(\lambda)=\sum_{k=0}^{2 m} a_{2 k} \lambda^{m-k}, p_{A_{\sigma}}(\lambda)=$ $\sum_{k=0}^{2 m} a_{2 k} \lambda^{k}$ are real polynomials.

Proof. By Proposition 4, we easily know that $a_{k}$ is a real number, and $a_{2 k+1}=0$. For any $k$, by Proposition 3, we have $B_{\sigma}^{2 k}=\left((B \widetilde{B})^{k}\right)_{\sigma} P_{n}$, so we can obtain the result (3).

2.3. On Solutions to the Quaternion j-Conjugate Matrix Equation $X-A \widehat{X} B=C Y$. In this subsection, we discuss the solution of the following quaternion matrix equation:

$$
X-A \widehat{X} B=C Y
$$

by means of real representation, where $A \in Q^{n \times n}, B \in Q^{p \times p}$, and $C \in Q^{n \times r}$ are known matrices, $X \in Q^{n \times p}$ and $Y \in Q^{r \times p}$ are unknown matrices.

We first define the real representation of quaternion matrix equation (17) by

$$
V-A_{\sigma} V B_{\sigma}=C_{\sigma} P_{r} W
$$

According to (1) in Proposition 3, the quaternion matrix equation (17) is equivalent to the following equation:

$$
(X-A \widehat{X} B)_{\sigma}=X_{\sigma}-A_{\sigma} X_{\sigma} B_{\sigma}
$$

Therefore, the matrix equation (17) can be converted into

$$
X_{\sigma}-A_{\sigma} X_{\sigma} B_{\sigma}=C_{\sigma} P_{r} Y_{\sigma}
$$

Thus, we have the following conclusion.

Proposition 6. Given the quaternion matrices $A \in Q^{n \times n}$, $B \in Q^{p \times p}$ and $C \in Q^{n \times r}$, then the quaternion matrix equation (17) has a solution $(X, Y)$ if and only if the real representation matrix equation (18) has a solution $(V, W)=\left(X_{\sigma}, Y_{\sigma}\right)$.

Theorem 7. Let $A \in Q^{n \times n}, B \in Q^{p \times p}$, and $C \in Q^{n \times r}$. Then quaternion matrix equation (17) has a solution $(X, Y)$ if and only if real representation matrix equation (18) has a solution $(V, W)$. Furthermore, if $(V, W)$ is a solution to (18), then the following quaternion matrices are solutions to quaternion matrix equation (17):

$$
\begin{aligned}
& X=\frac{1}{16}\left[\begin{array}{llll}
I_{n} & i I_{n} & j I_{n} & k I_{n}
\end{array}\right] \\
& \times\left(V-Q_{n}^{-1} V Q_{p}+R_{n}^{-1} V R_{p}-S_{n}^{-1} V S_{p}\right)\left[\begin{array}{c}
I_{p} \\
-i I_{p} \\
-j I_{p} \\
-k I_{p}
\end{array}\right], \\
& Y=\frac{1}{16}\left[\begin{array}{llll}
I_{r} & i I_{r} & j I_{r} & k I_{r}
\end{array}\right] \\
& \times\left(W-Q_{n}^{-1} W Q_{p}+R_{n}^{-1} W R_{p}-S_{n}^{-1} W S_{p}\right)\left[\begin{array}{c}
I_{p} \\
-i I_{p} \\
-j I_{p} \\
-k I_{p}
\end{array}\right] .
\end{aligned}
$$

Proof. By (3) of Proposition 3, the quaternion matrix equation (18) is equivalent to

$$
V-R_{n}^{-1} A_{\sigma} R_{n} V R_{p}^{-1} B_{\sigma} R_{p}=R_{n}^{-1} C_{\sigma} R_{r} P_{r} W .
$$

After multiplying the two sides of quaternion matrix equation (22) by $R_{p}^{-1}$, we can obtain

$$
V R_{p}^{-1}-R_{n}^{-1} A_{\sigma} R_{n} V R_{p}^{-1} B_{\sigma}=R_{n}^{-1} C_{\sigma} R_{r} P_{r} W R_{p}^{-1} .
$$

Before multiplying the two sides of quaternion matrix equation (23) by $R_{n}$, we have

$$
R_{n} V R_{p}^{-1}-A_{\sigma} R_{n} V R_{p}^{-1} B_{\sigma}=C_{\sigma} R_{r} P_{r} W R_{p}^{-1} .
$$

Noting that $R_{p}^{-1}=-R_{p}, R_{r} P_{r}=P_{r} R_{r}$, we give

$$
R_{n}^{-1} V R_{p}-A_{\sigma} R_{n}^{-1} V R_{p} B_{\sigma}=C_{\sigma} P_{r} R_{r}^{-1} W R_{p} .
$$

This shows that if $(V, W)$ is a real solution of matrix equation (18), then $\left(R_{n}^{-1} V R_{p}, R_{r}^{-1} W R_{p}\right)$ is also a real solution of quaternion matrix equation (18). In addition, according to (3) of Proposition 3, the quaternion matrix equation (18) is also equivalent to

$$
V-Q_{n} A_{\sigma} Q_{n} V Q_{p} B_{\sigma} Q_{p}=Q_{n} C_{\sigma} Q_{r} P_{r} W .
$$

After multiplying the two sides of quaternion matrix equation (26) by $Q_{p}^{-1}$, we have

$$
V Q_{p}^{-1}-Q_{n} A_{\sigma} Q_{n} V Q_{p} B_{\sigma}=Q_{n} C_{\sigma} Q_{r} P_{r} W Q_{p}^{-1}
$$

Noting that $Q_{p}^{-1}=-Q_{p}, Q_{r} P_{r}=-P_{r} Q_{r}$, before multiplying the two sides of the quaternion matrix equation (27) by $Q_{n}^{-1}$, gives

$$
\left(-Q_{n}^{-1} V Q_{p}\right)-A_{\sigma}\left(-Q_{n}^{-1} V Q_{p}\right) B_{\sigma}=C_{\sigma} P_{p}\left(-Q_{r}^{-1} W Q_{p}\right) .
$$


This is to say that if $(V, W)$ is a real solution of matrix equation (18), then $\left(-Q_{n}^{-1} V Q_{p},-Q_{r}^{-1} W Q_{p}\right)$ is also a real solution of matrix equation (18). Similarly, we can prove that $\left(-S_{n}^{-1} V S_{p},-S_{r}^{-1} W S_{p}\right)$ is also a real solution of quaternion matrix equation (18). In this case, the conclusion can be obtained along the line of the proof of Theorem 4.2 in [13].

Theorem 8. Given the quaternion matrices $A \in Q^{n \times n}, B \in$ $Q^{p \times p}$, and $C \in Q^{n \times r}$, let

$$
\begin{gathered}
f_{\left(I, A_{\sigma}\right)}(s)=\operatorname{det}\left(I_{4 n}-s A_{\sigma}\right)=\sum_{k=0}^{2 n} a_{2 k} s^{2 k}, \\
p_{A_{\sigma}}(s)=\sum_{k=0}^{2 n} a_{2 k} s^{k} .
\end{gathered}
$$

Then the matrices $X \in Q^{n \times p}, Y \in Q^{r \times p}$ are given by

$$
\begin{gathered}
X=\sum_{k=0}^{2 n-1} \sum_{s=k}^{2 n-1} \alpha_{2 k}(A \widehat{A})^{s-k} C Z(\widehat{B} B)^{s} \\
+\sum_{k=0}^{2 n-1} \sum_{s=k}^{2 n-1} \alpha_{2 k}(A \widehat{A})^{s-k} A \widehat{C Z} B(\widehat{B} B)^{s}, \\
Y=Z p_{A_{\sigma}}(\widehat{B} B),
\end{gathered}
$$

in which $Z$ is an arbitrary quaternion matrix.

Proof. If Yakubovich quaternion $j$-conjugate matrix equation (17) has solution $(X, Y)$, then real representation matrix equation (18) has solution $(V, W)=\left(X_{\sigma}, Y_{\sigma}\right)$ with the free parameter $Z_{\sigma}$. By Theorems 2 and 7 , we have

$$
\begin{aligned}
X_{\sigma}= & \sum_{k=0}^{2 n-1} \sum_{j=0}^{k} \alpha_{j} A_{\sigma}^{j-k} C_{\sigma} P_{r} Z_{\sigma} B_{\sigma}^{j} \\
= & \sum_{k=0}^{2 n-1} \sum_{j=2 k}^{4 n-1} \alpha_{2 k} A_{\sigma}^{j-2 k} C_{\sigma} P_{r} Z_{\sigma} B_{\sigma}^{j} \\
= & \sum_{k=0}^{2 n-1} \alpha_{2 k}\left[\sum_{s=k}^{2 n-1} A_{\sigma}^{2 s-2 k} C_{\sigma} P_{r} Z_{\sigma} B_{\sigma}^{2 s}\right. \\
= & \left.+\sum_{s=0}^{2 n-1} A_{\sigma}^{2 s-2 k+1} C_{\sigma} P_{r} Z_{\sigma} B_{\sigma}^{2 s+1}\right] \\
& \times\left[\sum_{s=k}^{2 n-1}\left((A \widehat{A})^{s-k}\right)_{\sigma} P_{n} C_{\sigma} P_{r} Z_{\sigma}\left((\widehat{B} B)^{s}\right)_{\sigma} P_{p}\right. \\
& \left.+\sum_{s=k}^{2 n-1}\left((A \widehat{A})^{s-k}\right)_{\sigma} P_{n} A_{\sigma} C_{\sigma} P_{r} Z_{\sigma} B_{\sigma}\left((\widehat{B} B)^{s}\right)_{\sigma} P_{p}\right]
\end{aligned}
$$

$$
\begin{aligned}
=\sum_{k=0}^{2 n-1} \alpha_{2 k}[ & \sum_{s=k}^{2 n-1}\left((A \widehat{A})^{s-k} C Z(\widehat{B} B)^{s}\right)_{\sigma} \\
& \left.+\sum_{s=k}^{2 n-1}\left((A \widehat{A})^{s-k} A \widehat{C} \widehat{Z} B(\widehat{B} B)^{s}\right)_{\sigma}\right] .
\end{aligned}
$$

In addition, by Proposition $5, f_{\left(I, A_{\sigma}\right)}(s)$ is a real polynomial and $f_{\left(I, A_{\sigma}\right)}\left(B_{\sigma}\right)=\left(p_{A_{\sigma}}(B \widehat{B})\right)_{\sigma} P_{p}$. So according to Proposition 3, we obtain

$$
Y_{\sigma}=Z_{\sigma} f_{\left(I, A_{\sigma}\right)}\left(B_{\sigma}\right)=Z_{\sigma}\left(p_{A_{\sigma}}(B \widehat{B})\right)_{\sigma} P_{p}=\left(Z p_{A_{\sigma}}(\widehat{B} B)\right)_{\sigma} .
$$

Thus, the conclusion above has been proved.

In the following, we provide an equivalent statement of Theorem 8 .

Theorem 9. Given quaternion matrices $A \in Q^{n \times n}, B \in Q^{p \times p}$, and $C \in Q^{n \times r}$, let

$$
\begin{gathered}
f_{\left(I, A_{\sigma}\right)}(s)=\operatorname{det}\left(I_{4 n}-s A_{\sigma}\right)=\sum_{k=0}^{2 n} a_{2 k} s^{2 k}, \\
p_{A_{\sigma}}(s)=\sum_{k=0}^{2 n} a_{2 k} s^{k} .
\end{gathered}
$$

Then the matrices $X \in Q^{n \times p}, Y \in Q^{r \times p}$ given by (30) have the following equivalent form:

$$
\begin{gathered}
X=Q_{c}(A \widehat{A}, C, 2 n) S_{r}\left(I, A_{\sigma}\right) Q_{o}(B \widehat{B}, Z, 2 n) \\
+Q_{c}(A \widehat{A}, A \widehat{C}, 2 n) S_{r}\left(I, A_{\sigma}\right) Q_{o}(\widehat{B} B, \widehat{Z} B, 2 n), \\
Y=Z p_{A_{\sigma}}(\widehat{B} B),
\end{gathered}
$$

in which $Z$ is an arbitrary quaternion matrix.

Proof. By the direct computation, we have

$$
\begin{aligned}
& \sum_{k=0}^{2 n-1} \sum_{s=k}^{2 n-1} \alpha_{2 k}(A \widehat{A})^{s-k} C Z(\widehat{B} B)^{s} \\
& =Q_{c}(A \widehat{A}, C, n) S_{r}\left(I, A_{\sigma}\right) Q_{o}(B \widehat{B}, Z, 2 n), \\
& \sum_{k=0}^{2 n-1} \sum_{s=k}^{2 n-1} \alpha_{2 k}(A \widehat{A})^{s-k} A \widehat{C} \widehat{Z} B(\widehat{B} B)^{s} \\
& =Q_{c}(A \widehat{A}, A \widehat{C}, 2 n) S_{r}\left(I, A_{\sigma}\right) Q_{o}(\widehat{B} B, \widehat{Z} B, 2 n) .
\end{aligned}
$$

Thus, the first conclusion has been proved. With this the second conclusion is obviously true.

Finally, we consider the solution to the so-called KalmanYakubovich $j$-conjugate quaternion matrix equation

$$
X-A \widehat{X} B=C .
$$

Based on the main result proposed above, we have the following conclusions regarding the matrix equation (36). 
Corollary 10. Given quaternion matrices $A \in Q^{n \times n}, B \in$ $Q^{p \times p}$, and $C \in Q^{n \times p}$, let

$$
\begin{gathered}
f_{\left(I, A_{\sigma}\right)}(s)=\operatorname{det}\left(I_{4 n}-s A_{\sigma}\right)=\sum_{k=0}^{2 n} a_{2 k} s^{2 k}, \\
p_{A_{\sigma}}(s)=\sum_{k=0}^{2 n} a_{2 k} s^{k} .
\end{gathered}
$$

If $X$ is a solution of equation (36), then

$$
\begin{aligned}
X p_{A_{\sigma}}(\widehat{B} B)= & \sum_{k=0}^{2 n-1} \sum_{j=k}^{2 n-1} \alpha_{2 k}(A \widehat{A})^{j-k} C(\widehat{B} B)^{j} \\
& +\sum_{k=0}^{2 n-1} \sum_{j=k}^{2 n-1}(A \widehat{A})^{j-k} A \widehat{C} B(\widehat{B} B)^{j} .
\end{aligned}
$$

Proof. If $X$ is a solution of equation (36), then $Y=X_{\sigma}$ is a solution of the equation $X_{\sigma}-A_{\sigma} X_{\sigma} B_{\sigma}=C_{\sigma}$. By Theorem 3 in [22] and Proposition 3, we have

$$
X_{\sigma} f_{\left(I, A_{\sigma}\right)}\left(B_{\sigma}\right)=\sum_{k=0}^{2 n-1} \sum_{j=2 k}^{4 n-1} \alpha_{2 k} A_{\sigma}^{j-2 k} C_{\sigma} B_{\sigma}^{j} .
$$

By Proposition 5, $f_{\left(I, A_{\sigma}\right)}(s)$ is a real polynomial and $f_{\left(I, A_{\sigma}\right)}\left(B_{\sigma}\right)=\left(p_{A_{\sigma}}(B \widehat{B})\right)_{\sigma} P_{p}$. So from Proposition 3 and (39), we have

$$
\begin{aligned}
& {\left[X p_{A_{\sigma}}(B \widehat{B})\right]_{\sigma}} \\
& =X_{\sigma}\left[p_{A_{\sigma}}(\widehat{B} B)\right]_{\sigma} P_{p} \\
& =X_{\sigma} f_{\left(I, A_{\sigma}\right)}\left(B_{\sigma}\right)=\sum_{k=0}^{2 n-1} \sum_{j=2 k}^{4 n-1} \alpha_{2 k} A_{\sigma}^{j-2 k} C_{\sigma} B_{\sigma}^{j} \\
& =\sum_{k=0}^{2 n-1} \alpha_{2 k}\left[\sum_{j=k}^{2 n-1} A_{\sigma}^{2 j-2 k} C_{\sigma} B_{\sigma}^{2 j}\right. \\
& \left.\quad+\sum_{j=k}^{2 n-1} A_{\sigma}^{2 j+1-2 k} C_{\sigma} B_{\sigma}^{2 j+1}\right] \\
& =\sum_{k=0}^{2 n-1} \alpha_{2 k}\left[\sum_{j=k}^{2 n-1}\left((A \widehat{A})^{j-k}\right)_{\sigma} P_{n} C_{\sigma}\left((\widehat{B} B)^{j}\right)_{\sigma} P_{p}\right. \\
& \left.\quad+\sum_{j=k}^{2 n-1}\left((A \widehat{A})^{j-k}\right)_{\sigma} P_{n} A_{\sigma} C_{\sigma} B_{\sigma}\left((\widehat{B} B)^{j}\right)_{\sigma} P_{p}\right]
\end{aligned}
$$

$$
\begin{aligned}
& =\sum_{k=0}^{2 n-1} \alpha_{2 k} \\
& \quad \times\left[\sum_{j=k}^{2 n-1}\left((A \widehat{A})^{j-k} C(\widehat{B} B)^{j}\right)_{\sigma}\right. \\
& \left.\left.+\sum_{k=0}^{2 n-1} \sum_{j=k}^{2 n-1} \alpha_{2 k}^{2 n-1}\left((A \widehat{A})^{j-k} C(\widehat{B} B)^{j}\right)_{\sigma}^{j-k} A \widehat{C} B(\widehat{B} B)^{j}\right)_{\sigma}\right] \\
& +\sum_{k=0}^{2 n-1} \sum_{j=k}^{2 n-1}\left((A \widehat{A})^{j-k} A \widehat{C} B(\widehat{B} B)^{j}\right)_{\sigma} .
\end{aligned}
$$

Thus, the first conclusion has been proved. With this the second conclusion is obviously true.

In the following, we provide an equivalent statement of Theorem 7.

Corollary 11. Given quaternion matrices $A \in Q^{n \times n}, B \in Q^{p \times p}$, and $C \in Q^{n \times p}$, let

$$
\begin{gathered}
f_{\left(I, A_{\sigma}\right)}(s)=\operatorname{det}\left(I_{4 n}-s A_{\sigma}\right)=\sum_{k=0}^{2 n} a_{2 k} s^{2 k}, \\
p_{A_{\sigma}}(s)=\sum_{k=0}^{2 n} a_{2 k} s^{k} .
\end{gathered}
$$

If $X$ is a solution of (36), then

$$
\begin{aligned}
X p_{A_{\sigma}}(\widehat{B} B)= & Q_{c}(A \widehat{A}, C, 2 n) S_{p}\left(I, A_{\sigma}\right) Q_{o}\left(\widehat{B} B, I_{p}, 2 n\right) \\
& +Q_{c}(A \widehat{A}, A, 2 n) S_{n}\left(I, A_{\sigma}\right) Q_{o}(\widehat{B} B, \widehat{C} B, 2 n) .
\end{aligned}
$$

\section{Complex Conjugate Matrix Equation}

$$
X-A \bar{X} B=C Y
$$

In this section, we study the solution to the complex matrix equation

$$
X-A \bar{X} B=C Y,
$$

where $A \in C^{n \times n}, B \in C^{p \times p}$, and $C \in C^{n \times r}$. Next, we define real representation of complex matrix as follows.

For any complex matrix $A=A_{1}+A_{2} i \in C^{m \times n}, A_{l} \in$ $R^{m \times n}(l=1,2$.$) , we define a real representation of a complex$ matrix as

$$
A_{\sigma}=\left[\begin{array}{cc}
A_{1} & A_{2} \\
A_{2} & -A_{1}
\end{array}\right] \in R^{2 m \times 2 n} .
$$


Then the real matrix $A_{\sigma}$ is called real representation of complex matrix $A$.

Let

$$
P_{t}=\left[\begin{array}{cc}
I_{t} & 0 \\
0 & -I_{t}
\end{array}\right], \quad Q_{t}=\left[\begin{array}{cc}
0 & I_{t} \\
-I_{t} & 0
\end{array}\right],
$$

in which $I_{t}$ is $t \times t$ identity matrix. Then $P_{t}, Q_{t}$ are unitary matrices. The real presentation has the following properties, which are given by Jiang and Wei [14].

Proposition 12. Consider the following.

(1) If $A, B \in C^{m \times n}, a \in R$, then $(A+B)_{\sigma}=A_{\sigma}+B_{\sigma}$, $(a A)_{\sigma}=a A_{\sigma}, P_{m} A_{\sigma} P_{n}=(\bar{A})_{\sigma}$;

(2) let $A \in C^{m \times n}, C \in C^{n \times s}, a \in R$, then $(A C)_{\sigma}=A_{\sigma} P_{n} C_{\sigma}$;

(3) if $A \in C^{m \times m}$, then complex matrix $A$ is nonsingular if and only if $A_{\sigma}$ is nonsingular;

(4) if $A \in C^{m \times m}$, then $A_{\sigma}^{2 k}=\left((A \bar{A})^{k}\right)_{\sigma} P_{m}$;

(5) if $A \in C^{m \times n}$, then $Q_{m} A_{\sigma} Q_{n}=A_{\sigma}$.

Actually, since complex matrix is a special case of quaternion matrix, in this case, we also have the following similar results. Because the proofs are similar to Section 2 and are omitted.

Theorem 13. Given complex matrices $A \in C^{n \times n}, B \in C^{p \times p}$, and $C \in C^{n \times r}$. Let

$$
\begin{gathered}
f_{\left(I, A_{\sigma}\right)}(s)=\operatorname{det}\left(I_{2 n}-s A_{\sigma}\right)=\sum_{k=0}^{n} a_{2 k} s^{2 k}, \\
p_{A_{\sigma}}(s)=\sum_{k=0}^{n} a_{2 k} s^{k} .
\end{gathered}
$$

Then the solution to the matrix equation (43) is given by

$$
\begin{gathered}
X=\sum_{k=0}^{n-1} \sum_{s=k}^{n-1} \alpha_{2 k}(A \bar{A})^{s-k} C Z(\bar{B} B)^{s} \\
+\sum_{k=0}^{n-1} \sum_{s=k}^{n-1} \alpha_{2 k}(A \bar{A})^{s-k} A \bar{C} \bar{Z} B(\bar{B} B)^{s}, \\
Y=Z p_{A_{\sigma}}(\bar{B} B) .
\end{gathered}
$$

In the following, we provide an equivalent statement of Theorem 13.

Theorem 14. Given complex matrices $A \in C^{n \times n}, B \in C^{p \times p}$, and $C \in C^{n \times p}$, let

$$
\begin{gathered}
f_{\left(I, A_{\sigma}\right)}(s)=\operatorname{det}\left(s I_{2 n}-A_{\sigma}\right)=\sum_{k=0}^{n} a_{2 k} s^{2 k}, \\
p_{A_{\sigma}}(s)=\sum_{k=0}^{n} a_{2 k} s^{k} .
\end{gathered}
$$

Then the matrices $X$ and $Y$ given by (47) have the following equivalent form:

$$
\begin{gathered}
X=Q_{c}(A \bar{A}, C, n) S_{r}\left(I, A_{\sigma}\right) Q_{o}(\bar{B} B, Z, n) \\
+Q_{c}(A \bar{A}, A \bar{C}, n) S_{r}\left(I, A_{\sigma}\right) Q_{o}(\bar{B} B, \bar{Z} B, n), \\
Y=Z P_{A_{\sigma}}(\bar{B} B) .
\end{gathered}
$$

Finally, we consider the solution to the so-called KalmanYakubovich-conjugate matrix

$$
X-A \bar{X} B=C .
$$

Based on the main result proposed above, we have the following conclusions regarding matrix equation (50).

Theorem 15. Given the complex matrices $A \in C^{n \times n}, B \in C^{p \times p}$, and $C \in C^{n \times p}$, let

$$
\begin{gathered}
f_{\left(I, A_{\sigma}\right)}(s)=\operatorname{det}\left(s I_{2 n}-A_{\sigma}\right)=\sum_{k=0}^{n} a_{2 k} s^{2 k}, \\
p_{A_{\sigma}}(s)=\sum_{k=0}^{n} a_{2 k} s^{k} .
\end{gathered}
$$

(1) If $X$ is a solution of (50), then

$$
\begin{aligned}
X p_{A_{\sigma}}(\bar{B} B)= & \sum_{k=0}^{n-1} \sum_{j=k}^{n-1} \alpha_{2 k}(A \bar{A})^{j-k} C(\bar{B} B)^{j} \\
& +\sum_{k=0}^{n-1} \sum_{j=k}^{n-1} \alpha_{2 k}(A \bar{A})^{j-k} A \bar{C} B(\bar{B} B)^{j} .
\end{aligned}
$$

(2) If $X$ is the unique solution of (50), then

$$
\begin{aligned}
X= & {\left[\sum_{k=0}^{n-1} \sum_{j=k}^{n-1} \alpha_{2 k}(A \bar{A})^{j-k} C(\bar{B} B)^{j}\right.} \\
& \left.+\sum_{k=0}^{n-1} \sum_{j=k}^{n-1} \alpha_{2 k}(A \bar{A})^{j-k} A \bar{C} B(\bar{B} B)^{j}\right] \\
& \times\left[p_{A_{\sigma}}(\bar{B} B)\right]^{-1} .
\end{aligned}
$$

Theorem 16. Given the complex matrices $A \in C^{n \times n}, B \in C^{p \times p}$, and $C \in C^{n \times p}$, let

$$
\begin{gathered}
f_{\left(I, A_{\sigma}\right)}(s)=\operatorname{det}\left(s I_{2 n}-A_{\sigma}\right)=\sum_{k=0}^{n} a_{2 k} s^{2 k}, \\
p_{A_{\sigma}}(s)=\sum_{k=0}^{n} a_{2 k} s^{k} .
\end{gathered}
$$

(1) If $X$ is a solution of (50), then

$$
\begin{aligned}
X p_{A_{\sigma}}(\bar{B} B)= & Q_{c}(A \bar{A}, C, n) S_{p}\left(I, A_{\sigma}\right) Q_{o}\left(\bar{B} B, I_{p}, n\right) \\
& +Q_{c}(A \bar{A}, A, n) S_{n}\left(I, A_{\sigma}\right) Q_{o}(\bar{B} B, \bar{C} B, n)
\end{aligned}
$$


(2) If $X$ is the unique solution of (50), then

$$
\begin{aligned}
X=[ & Q_{c}(A \bar{A}, C, n) S_{p}\left(I, A_{\sigma}\right) Q_{o}\left(\bar{B} B, I_{p}, n\right) \\
& \left.+Q_{c}(A \bar{A}, A, n) S_{n}\left(I, A_{\sigma}\right) Q_{o}(\bar{B} B, \bar{C} B, n)\right] \\
& \times\left[p_{A_{\sigma}}(\bar{B} B)\right]^{-1} .
\end{aligned}
$$

\section{Illustrative Example}

In this section, we give an example to obtain the solution of complex conjugate matrix equation $X-A \bar{X} B=C Y$.

Example 1. Consider Yakubovich-conjugate matrix equation in the form of (43) with the following parameters:

$$
\begin{gathered}
A=\left[\begin{array}{cc}
1+i & 2 i \\
4 & 0
\end{array}\right], \quad B=\left[\begin{array}{cc}
3 & 4+i \\
1 & -2 i
\end{array}\right], \\
C=\left[\begin{array}{cc}
3 & 2 i \\
2-i & 4
\end{array}\right] .
\end{gathered}
$$

According to the definition of real representation of a complex matrix, we have

$$
A_{\sigma}=\left[\begin{array}{cccc}
1 & 0 & 1 & 2 \\
4 & 0 & 0 & 0 \\
1 & 2 & -1 & 0 \\
0 & 0 & -4 & 0
\end{array}\right] .
$$

By some simple computations, we have

$$
\begin{aligned}
& f_{\left(I, A_{\sigma}\right)}(\lambda)=64 \lambda^{4}-2 \lambda^{2}+1 \text {, } \\
& p_{A_{\sigma}}(\lambda)=64 \lambda^{2}-2 \lambda+1, \\
& S_{2}\left(A_{\sigma}\right)=\left[\begin{array}{cc}
I_{2} & 2 I_{2} \\
0 & I_{2}
\end{array}\right], \quad I_{2}=\left[\begin{array}{ll}
1 & 0 \\
0 & 1
\end{array}\right], \\
& Q_{c}(A \bar{A}, C, 2)=\left[\begin{array}{cccc}
3 & 2 i & 8+18 i & -8-4 i \\
2-i & 4 & 4-28 i & 8-24 i
\end{array}\right] \text {, } \\
& Q_{o}(\bar{B} B, Z, 2)=\left[\begin{array}{cc}
1 & i \\
-1 & 1 \\
11+2 i & 9+3 i \\
-10+3 i & -2+6 i
\end{array}\right] \\
& Q_{c}(A \bar{A}, A \bar{C}, 2)=\left[\begin{array}{cccc}
1+7 i & 2+6 i & -30-2 i & -60+12 i \\
12 & -8 i & 32-72 i & -32+16 i
\end{array}\right] \text {, } \\
& Q_{o}(\bar{B} B, \bar{Z} B, 2)=\left[\begin{array}{cc}
3-i & 2+i \\
-2 & -4-3 i \\
42-9 i & 40-15 i \\
-32-15 i & -49-18 i
\end{array}\right]
\end{aligned}
$$

Choose

$$
Z=\left[\begin{array}{cc}
1 & i \\
-1 & 1
\end{array}\right]
$$

then it follows from Theorem 14 that the solution of (43) is

$$
\begin{gathered}
X=\left[\begin{array}{cc}
659+840 i & 1649+1118 i \\
1350-3683 i & 1611-4132 i
\end{array}\right], \\
Y=\left[\begin{array}{cc}
10603+2684 i & 12078-133 i \\
-9261+4026 i & -6843+8052 i
\end{array}\right] .
\end{gathered}
$$

\section{Conclusions}

In the present paper, by means of the real representation of a quaternion matrix, we study the quaternion matrix equation $X-A \widetilde{X} B=C Y$. Compared to our previous results [10], there are no requirements on the coefficient matrix $A$. Explicit solutions to this quaternion matrix equation are established by application of the real representation of a quaternion matrix. As a special case of quaternion $j$-conjugate matrix equation, complex conjugate matrix equation $X-A \bar{X} B=$ $C Y$ is also considered and the explicit solutions to complex conjugate are proposed. In addition, the equivalent forms of the explicit solutions are given.

\section{Conflict of Interests}

The authors declare that there is no conflict of interests regarding the publication of this paper.

\section{Acknowledgments}

The authors are very grateful to the anonymous reviewers and the editor for their helpful comments and suggestions which have helped us in improving the quality of this paper. This project is granted financial support from the NNSF (nos. $61374025,61174141,11171226,11301247)$ of China, the Postdoctoral Science Foundation of China (no. 2013M541900), the Research Awards Young and Middle-Aged Scientists of Shandong Province (BS2011SF009, BS2011DX019), and the Excellent Youth Foundation of Shandong's Natural Scientific Committee (JQ201219).

\section{References}

[1] R. R. Bitmead, "Explicit solutions of the discrete-time Lyapunov matrix equation and Kalman-Yakubovich equations," IEEE Transactions on Automatic Control, vol. 26, no. 6, pp. 1291-1294, 1981.

[2] B. H. Kwon and M. J. Youn, "Eigenvalue-generalized eigenvector assignment by output feedback," IEEE Transactions on Automatic Control, vol. 32, no. 5, pp. 417-421, 1987.

[3] D. G. Luenberger, "An introduction to observers," IEEE Transactions on Automatic Control, vol. 16, pp. 596-602, 1971.

[4] C.-C. Tsui, "New approach to robust observer design," International Journal of Control, vol. 47, no. 3, pp. 745-751, 1988.

[5] J. Chen, R. J. Patton, and H.-Y. Zhang, "Design of unknown input observers and robust fault detection filters," International Journal of Control, vol. 63, no. 1, pp. 85-105, 1996.

[6] J. Park and G. Rizzoni, "An eigenstructure assignment algorithm for the design of fault detection filters," IEEE Transactions on Automatic Control, vol. 39, no. 7, pp. 1521-1524, 1994. 
[7] S. Yuan and A. Liao, "Least squares solution of the quaternion matrix equation $X-A \widehat{X} B=C$ with the least norm," Linear and Multilinear Algebra, vol. 59, no. 9, pp. 985-998, 2011.

[8] S. F. Yuan, A. P. Liao, and G. Z. Yao, "The matrix nearness problem associated with the quaternion matrix equation $A X A^{H}+$ $B Y B^{H}=C$," Journal of Applied Mathematics and Computing, vol. 37, no. 1-2, pp. 133-144, 2011.

[9] C. Song and G. Chen, "On solutions of matrix equation $X F$ $A X=C$ and $X F-A \widetilde{X}=C$ over quaternion field," Journal of Applied Mathematics and Computing, vol. 37, no. 1-2, pp. 57-68, 2011.

[10] C. Q. Song, G. L. Chen, and X. D. Wang, "On solutions of quaternion matrix equations $X F-A X=B Y$ and $X F-A \widetilde{X}=$ BY," Acta Mathematica Scientia, vol. 32, no. 5, pp. 1967-1982, 2012.

[11] S. Ling, M. Wang, and M. Wei, "Hermitian tridiagonal solution with the least norm to quaternionic least squares problem," Computer Physics Communications, vol. 181, no. 3, pp. 481-488, 2010.

[12] M. H. Wang, M. S. Wei, and Y. Feng, "An iterative algorithm for least squares problem in quaternionic quantum theory," Computer Physics Communications, vol. 179, no. 4, pp. 203-207, 2008.

[13] T. S. Jiang and M. S. Wei, "On a solution of the quaternion matrix equation $X-A \widetilde{X} B=C$ and its application," Acta Mathematica Sinica, vol. 21, no. 3, pp. 483-490, 2005.

[14] T. Jiang and $M$. Wei, "On solutions of the matrix equations $X-A X B=C$ and $X-A \bar{X} B=C$," Linear Algebra and Its Applications, vol. 367, pp. 225-233, 2003.

[15] L. Huang, "The quaternion matrix equation $\sum A^{i} X B_{i}$, Acta Mathematica Sinica, vol. 14, no. 1, pp. 91-98, 1998.

[16] J. H. Bevis, F. J. Hall, and R. E. Hartwig, "Consimilarity and the matrix equation $A \bar{X}-X B=C$," in Current Trends in Matrix Theory, pp. 51-64, North-Holland, New York, NY, USA, 1987.

[17] J. H. Bevis, F. J. Hall, and R. E. Hartwig, "The matrix equation $A \bar{X}-X B=C$ and its special cases," SIAM Journal on Matrix Analysis and Applications, vol. 9, no. 3, pp. 348-359, 1988.

[18] B. Hanzon, "A faddeev sequence method for solving Lyapunov and Sylvester equations," Linear Algebra and Its Applications, vol. 241, pp. 401-430, 1996.

[19] A.-G. Wu, G.-R. Duan, and H.-H. Yu, "On solutions of the matrix equations $X F-A X=C$ and $X F-A \bar{X}=C$," Applied Mathematics and Computation, vol. 183, no. 2, pp. 932-941, 2006.

[20] A.-G. Wu, Y.-M. Fu, and G.-R. Duan, "On solutions of matrix equations $V-A V F=B W$ and $V-A \bar{V} F=B W$," Mathematical and Computer Modelling, vol. 47, no. 11-12, pp. 1181-1197, 2008.

[21] B. Hanzon and R. M. Peeters, "A Feddeev sequence method for solving Lyapunov and Sylvester equations," Linear Algebra and Its Applications, vol. 241-243, pp. 401-430, 1996.

[22] A.-G. Wu, H.-Q. Wang, and G.-R. Duan, "On matrix equations $X-A X F=C$ and $X-A \bar{X} F=C$," Journal of Computational and Applied Mathematics, vol. 230, no. 2, pp. 690-698, 2009. 


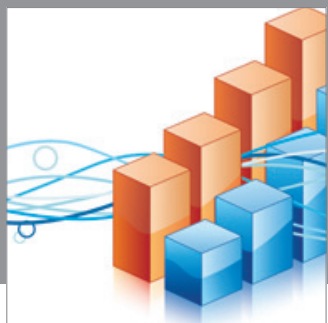

Advances in

Operations Research

mansans

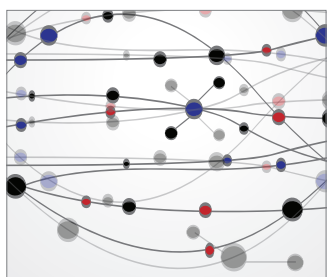

The Scientific World Journal
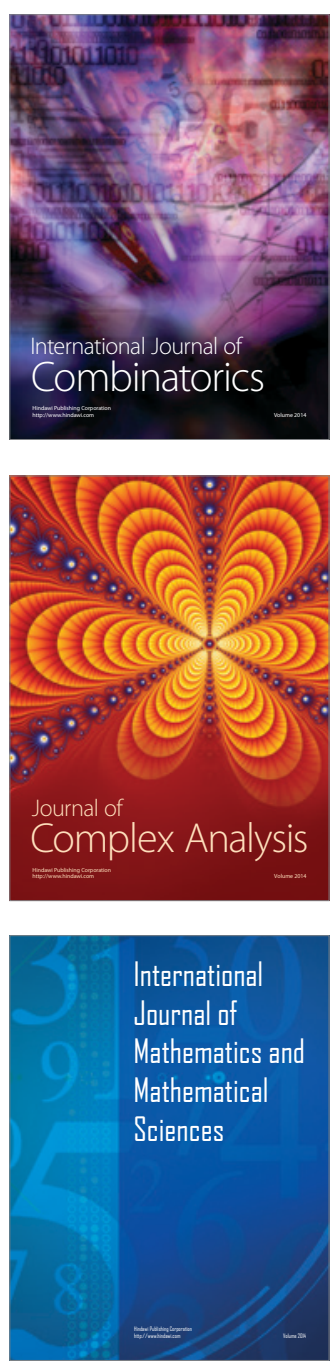
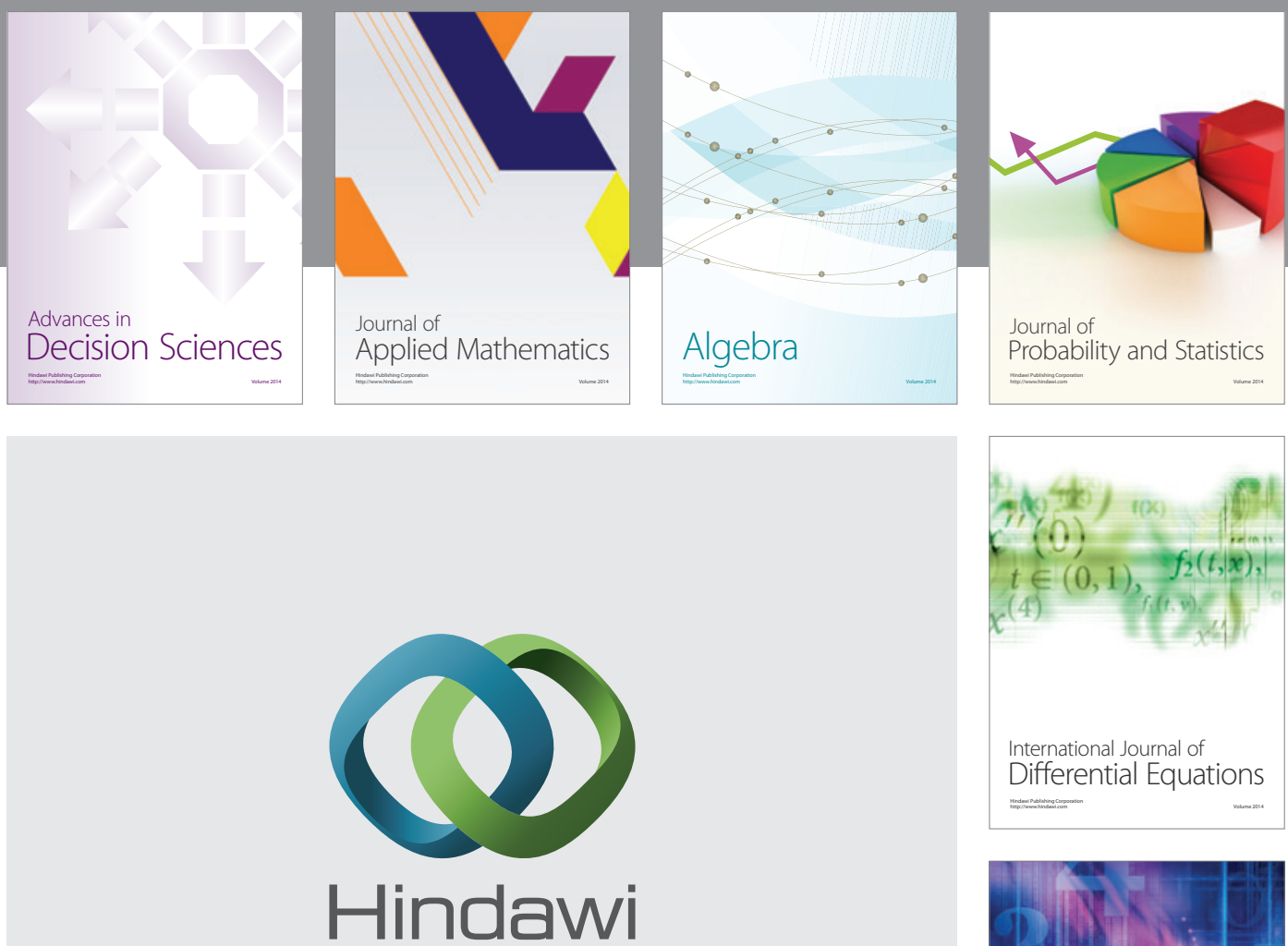

Submit your manuscripts at http://www.hindawi.com
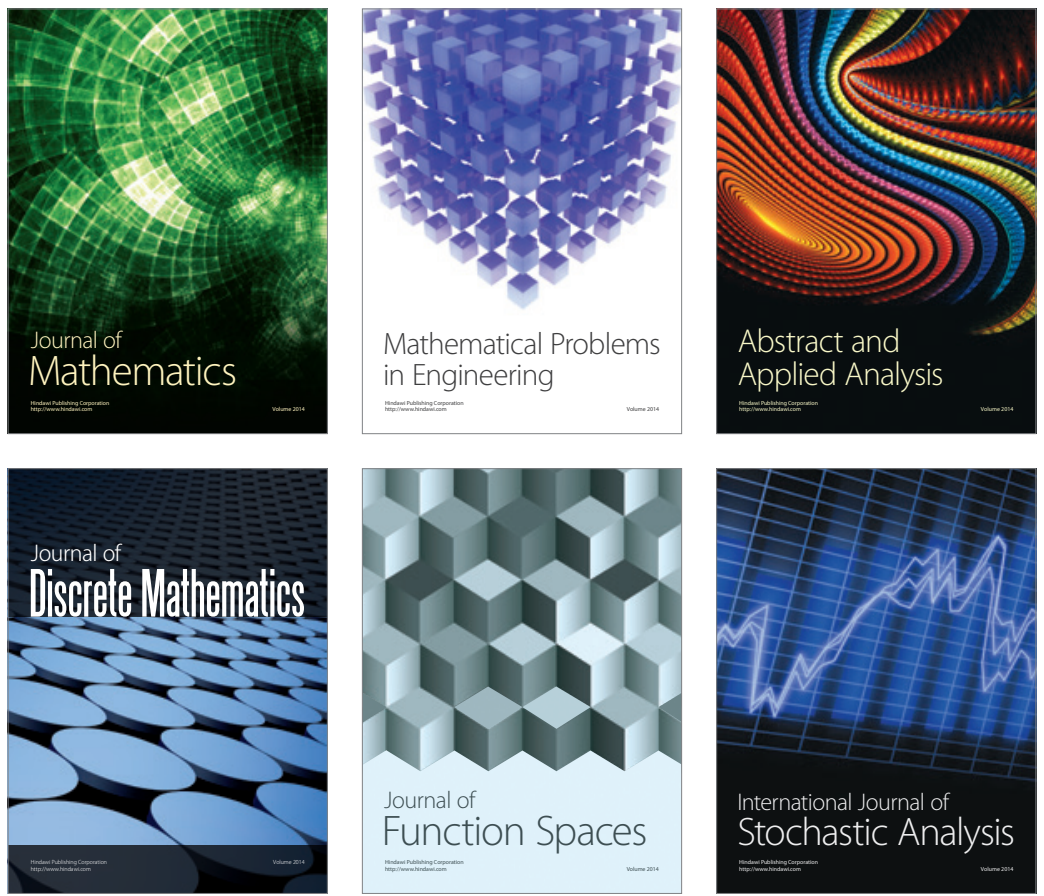

Journal of

Function Spaces

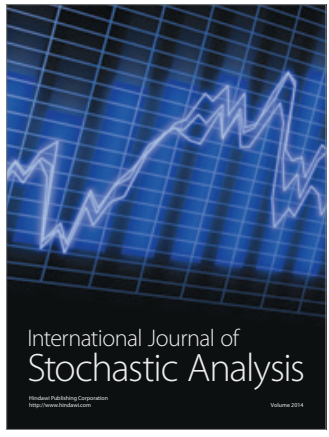

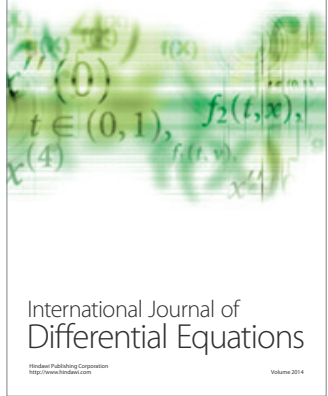
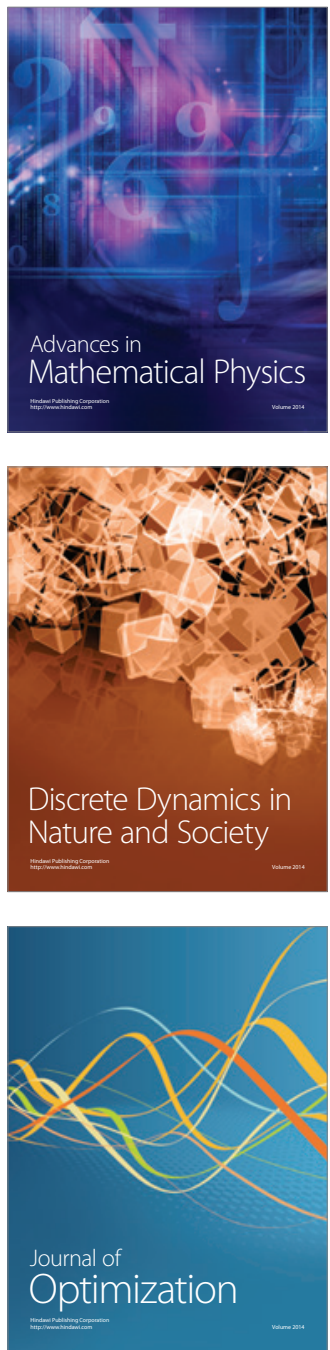